\title{
Application of Tunablef Self-collimation in LCD Photonic Crystals
}

\author{
Xiaohui $\mathrm{Li}^{1, \mathrm{a}^{*}}$, Huiyu Yang ${ }^{2, \mathrm{~b}}$ \\ ${ }^{1}$ College of electronic and information Engineering, Chongqing Radio And TV University, \\ No.1,Hualong Road, Jiulong District, Chongqing, China \\ ${ }^{2}$ CenterLab of Measurement \& Physical Test and Chemical Analysis in Chongqing Jianshe \\ Industry,Ltd.No.10,Xiejiawang Road, Jiulong District, Chongqing, China \\ a651739024@qq.com, ${ }^{\mathrm{b}} 461956904 @ q q . c o m$
}

\begin{abstract}
Keywords: tunability, photonic crystals, self-collimation, liquid crystals, self-collimation
\end{abstract}
\begin{abstract}
Photonic crystals with liquid-crystlal materials are extensively studied for their tunabilities. However, until now the studies mainly focused on the tunable photonic band gap properties. In this article, the tunable self-collimation in liquid-crystal-filled photonic crystals is discussed in detail. The impact of the twist angle of liquid crystals on the self-collimating frequencies is presented by using the plane wave expansion method. Moreover, the light propagation in the photonic crystal is simulated with the finite-difference time-domain method. The simulations show that the self-collimating frequencies are tunable by changing the liquid-crystal twist angle so that the self-collimating photonic crystals can be applied in different frequency bands.
\end{abstract}

\section{Introduction}

Liquid crystal is an anisotropic material, which is widely used in the design of various kinds of optical devices. When the liquid crystals are influenced by the electric field in a certain direction, the crystal column orientation will arrange along the applied electric field direction, so as to show the anisotropy. The crystal column orientation will change with the change of the applied electric field direction, and the dielectric constant also will change accordingly ${ }^{[1]}$.Consequently, the dielectric constant of liquid crystals can be controlled by adjusting the direction of the applied electric field. Exploiting the property of liquid crystals, when liquid crystal material is added into the photonic crystals, the property of photonic crystals can be adjusted using applied electric field. At present, the studies of liquid-crystal-filled photonic crystals mainly focused on the tunable photonic band gap properties $^{[2,3]}$. In additional, there are some studies employing on the tunable negative refraction ${ }^{[4,5]}$. In this paper, the tunable self-collimation in liquid-crystal-filled photonic crystal is discussed in detail.

Self-collimation is an anomalous dispersion phenomenon in photonic crystals, which is proposed firstly by Kosaka etal ${ }^{[6]}$. The group velocity of light waves in photonic crystals can be expressed as $\boldsymbol{v}_{g}=\nabla_{k} \omega(\boldsymbol{k})$, where $\mathrm{k}$ is wave vector, and $\mathrm{w}$ is angular frequency ${ }^{[7]}$. In the photonic crystals, the direction of light propagation is determined by the photonic crystals dispersion curve and it is perpendicular to the dispersion surface. The cross section of a given frequency dispersion curve is called the frequency line at the given frequency. The direction of group velocity and the frequency line are perpendicular to each other. When the frequency line is a straight line, light wave with the corresponding frequency will transmit without diffraction along the direction perpendicular to the frequency line, and in the process of transmission, the beam width is almost no change. The transmission is significantly different from the light propagation depending on the photonic band gap in photonic crystal waveguide. This phenomenon is called self-collimating effect of photonic crystals. In the photonic crystals, self-collimating transmission has some obvious advantages compared with waveguide transmission, such as: no defects, reducing the difficulty of processing; achieving the lower refractive index of the medium than the band gap formation required; self-collimating transmission in the case of misalignment without certain the coupling position and so on. 
Generally speaking, the self-collimating frequency of photonic crystal is fixed. In this paper, through the introduction of liquid crystal material, the self-collimating frequencies of photonic crystals are tunable by adjusting the direction of applied electric field.

\section{The Oretical Model of Lcd Photonic Crystal}

The liquid crystal columns will arrange along the direction of the applied electric field when the nematic liquid crystal is influenced by the applied electric field, which shows the anisotropy. In anisotropic medium, the propagation of electromagnetic wave still satisfies the wave equation ${ }^{[8] .}$

$$
\nabla \times\left(\frac{1}{\varepsilon(\mathbf{r})} \nabla \times \mathbf{H}(\mathbf{r})\right)=\left(\frac{\omega}{c}\right)^{2} \mathbf{H}(\mathbf{r})
$$

According to the Bloch theorem, the electromagnetic wave in the medium period can be expressed as the superposition of a series of plane wave. Therefore, magnetic field $\mathrm{H}(\mathrm{r})$ can be expressed as

$$
\mathbf{H}(\mathbf{r})=\sum_{\mathbf{G}} h(\mathbf{G}) \mathbf{e}_{\mathbf{G}} e^{\mathrm{i}(\mathbf{k}+\mathbf{G}) \mathbf{r}}
$$

In the periodic medium, dielectric tensor is a periodic function with a lattice vector $\mathrm{R}$. It satisfies $\varepsilon(\mathbf{r})=\varepsilon(\mathbf{r}+\mathbf{R})$. Fourier expansion is made in the reciprocal lattice vector space, we can get

$$
\varepsilon(\mathbf{r})=\sum_{\mathbf{G}} \varepsilon(\mathbf{G}) e^{\mathrm{i} \mathbf{G g}}
$$

For two-dimensional photonic crystals which arranged periodically in $x-y$ plane, the formula can be written as

$$
\varepsilon_{i j}(\mathbf{r})=\sum_{\mathbf{G}} \varepsilon_{i j}(\mathbf{G}) e^{\mathrm{i} \mathbf{G g}} \quad(i, j=x, y)
$$

The dielectric constant of the uniaxial crystal material is a tensor. In general, the intersection angle between optical axis of liquid crystal and $x-y$ plane is $\theta$, and the angle between the projection in $x-y$ plane and the $\mathrm{x}$ axis is $\varphi$. Them can be seen in fig.(1).

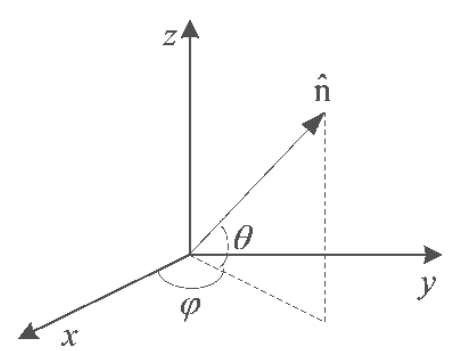

Fig. (1). Schematic diagram of the liquid crystal optical axis

When the liquid crystal optical axis is in $\mathrm{x}-\mathrm{y}$ plane, namely, $\theta$ is 0 , the dielectric constant tensor can be written as ${ }^{[9]}$ : 


$$
\left\{\begin{array}{l}
\varepsilon_{x x}=\varepsilon^{o} \sin ^{2} \varphi+\varepsilon^{e} \cos ^{2} \varphi \\
\varepsilon_{y y}=\varepsilon^{o} \cos ^{2} \varphi+\varepsilon^{e} \sin ^{2} \varphi \\
\varepsilon_{z z}=\varepsilon^{o} \\
\varepsilon_{x y}=\varepsilon_{y x}=\left(\varepsilon^{e}-\varepsilon^{o}\right) \sin \varphi \cos \varphi \\
\varepsilon_{x z}=\varepsilon_{z x}=0 \\
\varepsilon_{y z}=\varepsilon_{z y}=0
\end{array}\right.
$$

Where $\varepsilon 0$ and $\varepsilon e$ are respectively the unusual dielectric constant and the anomalous dielectric constant of liquid crystals (they are respectively $n_{o}=\sqrt{\varepsilon^{o} / \varepsilon_{0}}$ and $n_{e}=\sqrt{\varepsilon^{e} / \varepsilon_{0}}$ corresponding to the ordinary refractive index and the anomalous refractive index).

Putting formula (2), (3), (5) into (1) and multiplying by eG, we can get

$$
\sum_{\mathbf{G}^{\prime}} \mathbf{H}_{\mathbf{G}, \mathbf{G}^{\prime}} h\left(\mathbf{G}^{\prime}\right)=\left(\frac{\omega}{c}\right)^{2} h(\mathbf{G})
$$

For the two-dimensional problem, assuming that the photonic crystals arrange periodically in the $\mathrm{x}-\mathrm{y}$ plane and axial direction of dielectric cylinder or air hole is along the $\mathrm{z}$ direction, the above formula can be decomposed into TE mode and TM mode. The formula can be solved by solving the two independent problems. In this case, equation (6) can be expressed as follows.

$$
\begin{aligned}
& \sum_{\mathbf{G}^{\prime}}|\mathbf{k}+\mathbf{G}|\left|\mathbf{k}+\mathbf{G}^{\prime}\right| \times\left[\begin{array}{l}
e_{\mathbf{G}}^{2} g \varepsilon^{-1}\left(\mathbf{G}-\mathbf{G}^{\prime}\right) \mathfrak{g}_{\mathbf{G}}^{2}, \\
-e_{\mathbf{G}}^{1} g \varepsilon^{-1}\left(\mathbf{G}-\mathbf{G}^{\prime}\right) \mathcal{g}_{\mathbf{G}}^{2},
\end{array}\right. \\
& \left.\begin{array}{l}
-e_{\mathbf{G}}^{2} g \varepsilon^{-1}\left(\mathbf{G}-\mathbf{G}^{\prime}\right) \boldsymbol{g}_{\mathbf{G}}^{1} \\
e_{\mathbf{G}}^{1} \boldsymbol{g} \xi^{-1}\left(\mathbf{G}-\mathbf{G}^{\prime}\right) \boldsymbol{g}_{\mathbf{G}}^{1}
\end{array}\right] \times\left[\begin{array}{l}
h^{1}\left(\mathbf{G}^{\prime}\right) \\
h^{2}\left(\mathbf{G}^{\prime}\right)
\end{array}\right]=\left(\frac{\omega}{c}\right)^{2}\left[\begin{array}{l}
h^{1}(\mathbf{G}) \\
h^{2}(\mathbf{G})
\end{array}\right]
\end{aligned}
$$

The above equation is an infinite matrix eigenvalue problem, where $k$ is plane wave

vector, $h^{\lambda}(\mathbf{G})$ and $\mathbf{e}_{\mathbf{G}}^{\lambda}$ respectively are the amplitude and the unit vector of the polarization vector perpendicular to $(\mathrm{k}+\mathrm{G})$. In actual operation, we can select the appropriate number of plane waves to solve it numerically as needed. For general two-dimensional photonic band gap problem, when taking 441 plane waves, the error is less than $1 \%{ }^{[10]}$.

\section{Simulation and Analysis Tunable Self-Collimation}

Punching in the dielectric plate taking silicon $(n=3.50)$ as the background material according to tetragonal lattice periodically and filling with liquid crystals in the air holes can form liquid-crystal-filled photonic crystals. In order to verify the transmission of self-collimating beam in a long distance, the number of air holes is $54 \times 23$. The radius of air hole is $r=0.40 a$, where a is the lattice constant. Firstly, assume that photonic crystals arrange periodically in the $x-y$ plane, that is to say, the axial direction of air hole of photonic crystals is along $\mathrm{z}$ direction. And then assume that the pointing vectors of liquid crystals respectively are parallel and perpendicular to the $\mathrm{x}-\mathrm{y}$ plane for TE mode and TM mode.

For tunable liquid crystals, the common types of liquid crystal materials are 5CB, E7 and Phenylacetylene. Among them, Phenylacetylene is a new type of liquid crystals, which is firstly discovered by Fujisawagn and Sekine et al. A characteristic of the liquid crystals is the larger 
refractivity difference. their unusual and abnormal refractive indexes respectively are 1.590 and 2.223, and refractivity difference is 0.663 , which is further lager than the difference of refractive indexes of 5CB and E7 ${ }^{[11]}$. Therefore, the tunability of Phenylacetylene is larger than 5CB's and E7's. In this paper, Phenylacetylene liquid crystals will be chosen.

Adjusting the direction of applied electric field can control the twist angle of liquid crystals and then change the refractivity. Due to the change of refractivity, self-collimating frequencies of photonic crystals will change. In order to study the twist angle $\varphi$ of liquid crystals how to affect the self-collimation, to use the plane wave expansion method to calculate the frequency lines at different twist angles and achieve self-collimating frequencies from the corresponding frequency lines so that the relationship can be get between the twist angles of liquid crystals and self-collimating frequencies.

Next the study takes TE mode for instance and the method of TM mode is similar to it. The relationship between the twist angles of liquid crystals and self-collimating frequencies by using the plane wave expansion method to calculate is shown in fig.(2). In order to ensure the accuracy of calculation, each time all is calculated by using the 4096 plane wave. From the fig.(2), the self-collimating frequencies can be decreased gradually with the increase of the twist angles of liquid crystals for TE mode.

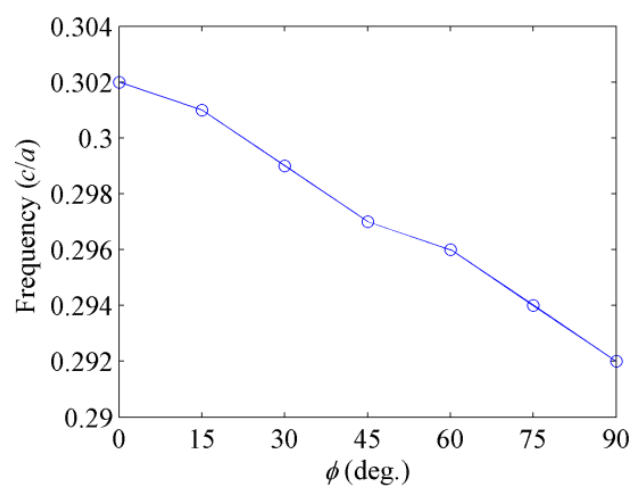

Fig.(2). The relationship between the twist angles of liquid crystals and self-collimating frequencies (TE mode)

In particular, when the twist angles of liquid crystals $\varphi$ equals $0^{\circ}$ and $90^{\circ}$, their frequency lines respectively are shown in figure $3(\mathrm{a})$ and (b). We can see that when the twist angle $\varphi=0^{\circ}$, self-collimating frequency fsc $=0.302 \mathrm{c} / \mathrm{a}$. And when the twist angle $\varphi=90^{\circ}$, self-collimating frequency fsc $=0.292 \mathrm{c} / \mathrm{a}$.

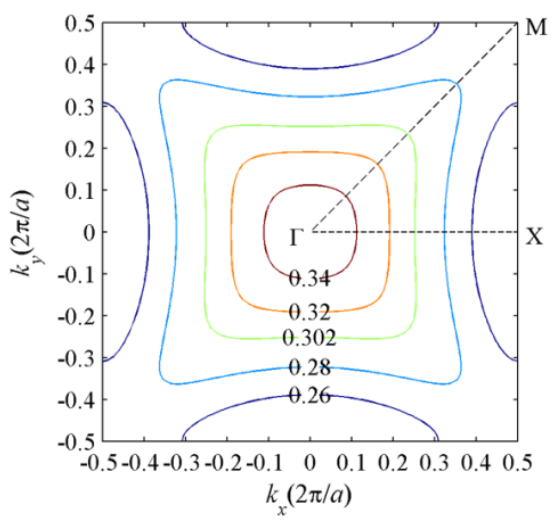

(a) $\varphi=0^{\circ}$ 


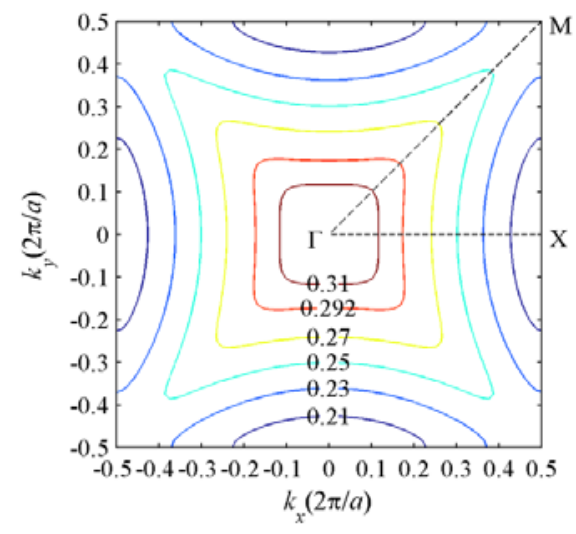

(b) $\varphi=90^{\circ}$

Fig.(3).The second band frequency line of photonic crystals for TE mode when $\varphi=0^{\circ}$ and $\varphi=90^{\circ}$

In order to verify the self-collimating tunability, the light propagation in the different twist angles of liquid crystals is simulated with the finite-difference time-domain method. The simulation software is FDTD and MEEP, which are open source and free [12]. Boundary conditions of FDTD employ the PML absorbing boundary conditions and the resolution ration of simulation is $\mathrm{a} / 32$. The gauss line source whose frequencies fsc are $0.302 \mathrm{c} / \mathrm{a}$ and $0.292 \mathrm{c} / \mathrm{a}$ respectively and spatial width is $4 \mathrm{a}$ projects from the left side of photonic crystals.

Fig.(4)(a) and (b) show the steady-state $\mathrm{Hz}$ field distribution of TE mode Gaussian beam propagation in photonic crystals when the twist angles of liquid crystals $\varphi$ are $0^{\circ}$ and $90^{\circ}$ respectively and frequency $\mathrm{f}$ is $0.302 \mathrm{c} / \mathrm{a}$. As is shown in figure 4 , we can see that beams almost have no diffraction propagation along a straight line in photonic crystals and there is almost no change for the beam width when the twist angle of liquid crystals $\varphi$ is $0^{\circ}$. Beams reflect a very good performance on self-collimating transmission. However, when the twist angle of liquid crystals $\varphi$ is $90^{\circ}$, beams have large divergence in photonic crystals, which does not meet the requirement of self-collimating transmission.

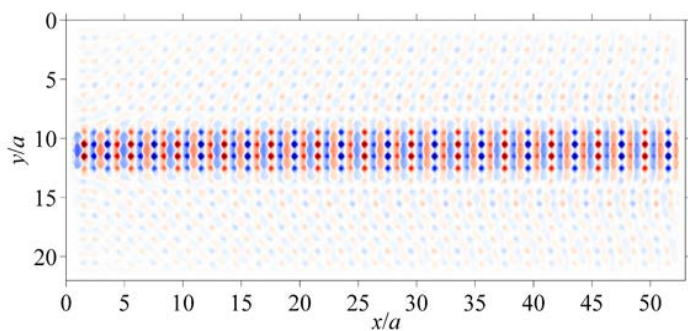

(a) $\varphi=0^{\circ}$

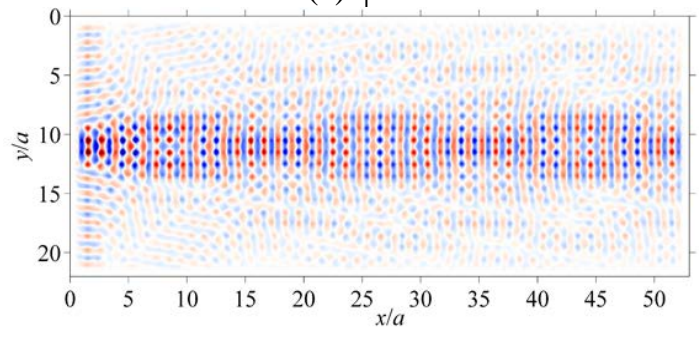

(b) $\varphi=90^{\circ}$

Fig.(4). The steady-state Hz field distribution of FDTD simulating beam propagation in photonic crystals $(\mathrm{f}=0.302 \mathrm{c} / \mathrm{a})$

On the contrary, as is shown in fig.(5)(b), when frequency of TE mode Gaussian beams satisfies $\mathrm{f}=0.292 \mathrm{c} / \mathrm{a}$ and the twist angle of liquid crystals $\varphi$ is $90^{\circ}$, beams have a good transmission characteristic on self-collimation. However, from the fig.(5)(a), beams have large divergence when the twist angle of liquid crystals $\varphi$ is $0^{\circ}$. 


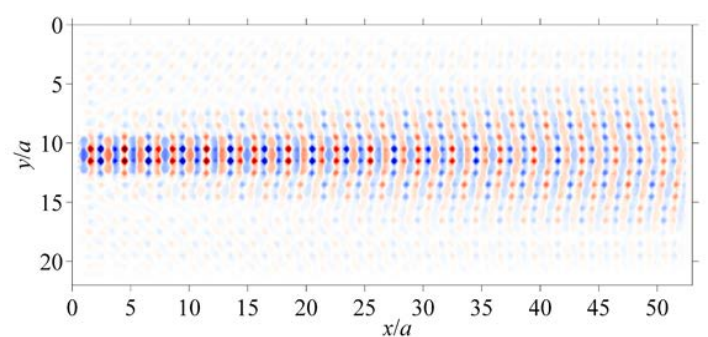

(a) $\varphi=0^{\circ}$

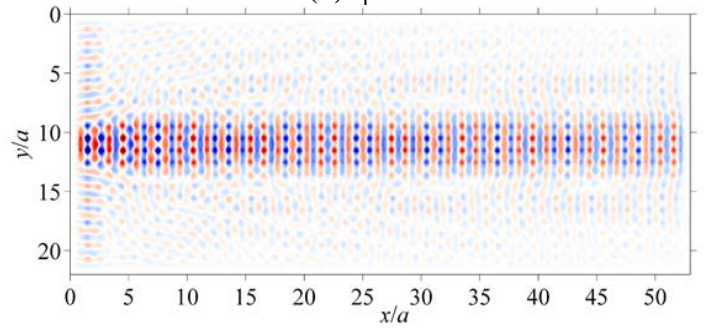

(b) $\varphi=90^{\circ}$

Fig.(5). The steady-state Hz field distribution of FDTD simulating beam propagation in photonic crystals $(\mathrm{f}=0.292 \mathrm{c} / \mathrm{a})$

The above simulation proved that self-collimating frequency of photonic crystals satisfies fsc $=0.302 \mathrm{c} / \mathrm{a}$ when the twist angle of liquid crystals $\varphi$ is $0^{\circ}$, and self-collimating frequency fsc is $0.292 \mathrm{c} / \mathrm{a}$ when $\varphi=90^{\circ}$. Therefore, self-collimating frequencies of photonic crystals are tunable by adjusting the applied electric field.

\section{Conclusion}

The tunable self-collimation in liquid-crystal-filled photonic crystals is discussed in detail. The impact of the twist angles of liquid crystals on the self-collimating frequencies is presented by using the plane wave expansion method. Moreover, the light propagation in the photonic crystals is simulated with the different twist angles in FDTD[13,14]. The research results show that the self-collimating frequencies are tunable by adjusting the direction of the applied electric field and then controlling the liquid-crystal twist angles so that the self-collimating photonic crystals can be applied in different frequency bands to meet the requirement of different occasions.

\section{Conflict of Interest}

We declare that we have no financial and personal relationships with other people or organizations that can inappropriately influence our work.

\section{Acknowledgements}

This research supported by the project "Research on negative refraction photonic crystal and its application” in Commission of Education of Chongqing,China(Grant No.KJ 131604).

\section{References}

[1] Jianling Yin, Xuguang Huang, Songhao Liu. Photonic Crystal Field-Sensitive Polarizer and Switch Modulated by Bemaic Liquid Crystals[J].Acta Physica Sinica,2006,55(10): 5268-5276.

[2] K. Busch, S. John. Liquid-crystal photonic-band-gap materials: The tunable electromagnetic vacuum [J]. Phys. Rev. Lett., 1999, 83 (5):967-970.

[3] S. W. Leonard, J. P. Mondia, H. M. van Driel, et al. Tunable two-dimensional photonic crystals using liquid-crystal infiltration [J]. Phys. Rev. B, 2000, 61 (4):R2389-R2392. 
[4] C. Y. Liu, N. W. Zhang, H. W. Wang, et al. Efficient tunable negative refraction photonic crystal achieved by an elliptic rod lattice with a nematic liquid crystal [J]. Physica B-Condensed Matter, 2009, 404 (21): 4060-4070.

[5] Y. Y. Wang, L. W. Chen. Tunable negative refraction photonic crystals achieved by liquid crystals [J]. Opt. Express, 2006, 14 (22):10580-10587.

[6] H. Kosaka, T. Kawashima, A. Tomita, et al. Self-collimating phenomena in photonic crystals [J]. Appl. Phys. Lett., 1999, 74 (9):1212-1214.

[7] D. W. Prather, S. Y. Shi, J. Murakowski, et al. Self-collimation in photonic crystal structures: a new paradigm for applications and device development [J]. J. Phys. D. Appl. Phys., 2007, 40 (9):2635-2651.

[8] J. D. Joannopoulos, S. G. Johnson, J. N. Winn, et al. Photonic crystals: modeling the flow of light. Princeton University Press, 2008.

[9] E. P. Kosmidou, E. E. Kriezis, T. D. Tsiboukis. Analysis of tunable photonic crystal devices comprising liquid crystal materials as defects [J]. IEEE. J. Quantum. Elect, 2005, 41 (5):657-665.

[10] C. Y. Liu, L. W. Chen. Tunable band gap in a photonic crystal modulated by a nematic liquid crystal [J]. Phys. Rev. B, 2005, 72 (4): 1331-1336.

[11] C. Sekine, K. Iwakura, N. Konya, et al. Synthesis and properties of some novel high birefringence phenylacetylene liquid crystal materials with lateral substituents [J]. Liq. Cryst., 2001, 28 (9):1375-1387.

[12] A. F. Oskooi, D. Roundy, M. Ibanescu, et al. MEEP: A flexible free-software package for electromagnetic simulations by the FDTD method [J]. Comput. Phys. Commun., 2010, 181 (3):687-702.

[13] Heming Chen,Xiaoying Wei. The Design of High-speed Photonic Crystal Optical Switch [J]. Opto-Electronic Engineering,2013(11): 34-39.

[14] Jihuan Pan,An Su,Chengju Meng. Modulation of Optical Thickness on Transmission Spectrum of Photonic Crystal [J]. Laser \& Infrared,2014(05): 559-562. 